\title{
Temperature dependence of water dynamics at an aqueous micellar surface: Atomistic molecular dynamics simulation studies of a complex system
}

\author{
Subrata Pal \\ Solid State and Structural Chemistry Unit, Indian Institute of Science, Bangalore 560012, India \\ Sundaram Balasubramanian* \\ Chemistry and Physics of Materials Unit, Jawaharlal Nehru Centre for Advanced Scientific Research, \\ Jakkur, Bangalore 560064, India \\ Biman Bagchi^ \\ Solid State and Structural Chemistry Unit, Indian Institute of Science, Bangalore 560012, India
}

\begin{abstract}
In order to study the temperature dependence of water dynamics at the surface of a self-organized assembly, we perform long atomistic molecular dynamics simulations of a micelle of cesium pentadecafluorooctanoate in water at two different temperatures, 300 and $350 \mathrm{~K}$. Since this micellar system is stable over a range of temperature, a detailed study of the microscopic dynamics of water at the surface of the micelle at both temperatures could be performed. The diffusion and dipolar orientational correlation function of the water molecules and the polar solvation dynamics of cesium ions at the micellar surface are calculated as a function of their location from the micellar surface. Our study reveals a strong temperature dependence. The relaxation of both the time correlation functions are highly nonexponential, and become very slow at $300 \mathrm{~K}$. It is found that while the slowness in the orientational time correlation function originates partly from the formation of bridge hydrogen bonds between the polar head groups $(\mathrm{PHG})$ of the micelle and the water molecules, the solvation dynamics slows down primarily due to the interaction of the positive cesium ions with the negatively charged PHGs.
\end{abstract}

\section{INTRODUCTION}

Self-organized molecular assemblies are usually found in nature in aqueous environments. These systems are stabilized by the hydrophobic interactions of the nonpolar tail and the hydrogen bonding of the polar head groups with the water molecules. In these systems, the water molecules that are often confined within (as in reverse micelles) or at the surface (as in micelles) of such assemblies have properties which are quite different from those in bulk water. These differences are in fact used in nature to carry out specific physico-chemical functions. ${ }^{1,2}$ The dynamics of such water molecules has recently been studied by using many different techniques, including dielectric relaxation, ${ }^{3}$ solvation dynamics, ${ }^{4-7}$ NMR relaxation dispersion (NMRD) ${ }^{8}$ and intermolecular water-solute NOE studies. ${ }^{9}$ These studies have led to the surprising discovery of the existence of a second, very slow, component in the dynamics of water in such restricted environments. ${ }^{10}$ This second component was found to decay in the hundreds to thousands of picoseconds. The first component remains ultrafast, similar to that in bulk water. The slow second component appears to be unique to water in complex systems and may play an important role in many chemical reactions such as electron transfer. ${ }^{11}$ The ori-

*Electronic mail: bala@jncasr.ac.in

^Electronic mail: bbagchi@sscu.iisc.ernet.in gin of this slow component has been attributed to the existence of a dynamic equilibrium between the "quasibound" and the free water molecules. ${ }^{2,12,13}$

Recently, we have presented atomistic molecular dynamics simulation studies of solvation ${ }^{14}$ and orientational ${ }^{15} \mathrm{dy}$ namics at the surface of an aqueous micelle at $350 \mathrm{~K}$. The micelle simulated was pentadecafluorooctanoate, with cesium being the counterion, commonly referred to as CsPFO. The system consisted of $62 \mathrm{CsPFO}$ and 10562 water molecules. The simulations were carried out for over $5 \mathrm{~ns}$. The results revealed the existence of a slow component, both in orientational dynamics of water and in the solvation time correlation function of the $\mathrm{Cs}^{+}$ions at the surface of the micelle. It was found that while the slowness in the orientational relaxation was due to the bridge hydrogen bonding of water molecules with the polar head groups of the micelle, the slowness of the solvation time correlation function is not due to the slow water molecules. It is due rather to the interaction of the $\mathrm{Cs}^{+}$ions with the polar head groups. Thus, the polar head groups of the surfactant molecules were found to play a very important role in the dynamics of both water and of ions at the micellar surface.

Because of the important role of soft interactions in their stability, the properties of self-organized assemblies are expected to exhibit strong temperature dependence. In the present study, we have compared the orientational and solvation dynamics at two different temperatures, 300 and $350 \mathrm{~K}$. 
Thus, we have performed further atomistic molecular dynamics (MD) simulations at $300 \mathrm{~K}$. The results of this comparison are really interesting. Not only do the two correlation functions display strong temperature dependence, we find that the solvation time correlation function becomes very slow at $300 \mathrm{~K}$. We attribute this slowdown to the increased stability of the quasibound water molecules due to the hydrogen bonding with the polar head groups.

It is important to realize that the water molecules are not permanently bound to the micellar surface, and are rather transient to the surface. This aspect raises several interesting issues regarding the role of averaging in the observable properties. Thus, single particle and collective properties could behave differently, as the collective properties, like dielectric relaxation and solvation dynamics of the system, are sensitive to the fraction of (slow) water molecules at the surface. Single particle properties can be investigated in solutions by following trajectories of the individual water molecules.

In the next section, we discuss the simulation details. In Sec. III, we present the results and discuss their significance. Section IV contains the concluding remarks.

\section{SIMULATION DETAILS}

As already mentioned, the surfactant is pentadecafluorooctanoate, with cesium being the counterion. The CsPFO- $\mathrm{H}_{2} \mathrm{O}$ system has been well studied experimentally $^{16,17}$ and is regarded as a typical binary to exhibit micellization. ${ }^{18}$ The amphiphiles are believed to form diskshaped (oblate ellipsoid) micelles, stable over an extensive range of concentration and temperature. As a function of concentration, the system exhibits isotropic, nematic, and lamellar phases. In the nematic phase, the mesogenic units are the individual micelles. The critical micelle concentration is around 0.02 weight fraction of CsPFO in water. At $350 \mathrm{~K}$, the isotropic phase is stable up to a weight fraction of 0.62 and the average aggregation number is around 60 .

The molecular dynamics simulations were carried out in the $N V T$ ensemble for an aggregate of $62 \mathrm{CsPFO}$ molecules in 10562 water molecules, i.e., at a weight fraction of 0.15 . The initial configuration of the micelle was built to mimic experimental data pertaining to its size and shape. ${ }^{17}$ This configuration, for the micelle with linear dimensions of 45 , $45,25 \AA$ along its principal axes, was placed in a box of water with linear dimensions of $84,84,55 \AA$. Water molecules in hard contact with the micelle were removed by observation. The counterions were placed at random positions among the water molecules with the constraint that they were at least $7 \AA$ away from the micellar head groups. The potential for water molecules is the extended simple point charge $(\mathrm{SPC} / \mathrm{E})$ model; ${ }^{19}$ the counterions carry a unit positive charge, which is compensated by a $+0.4 e$ charge on the carbon of the octanoate head group and a $-0.7 e$ charge on each of the oxygens of the head group. ${ }^{20}$ The surfactant is modeled with explicit fluorine atoms, and interactions between the fluorocarbon tails were obtained from the work of Sprik et al. on polytetrafluoroethylene. ${ }^{21}$ Other details of the potential parameters are provided elsewhere. ${ }^{14}$ The equations of motion were integrated with the reversible reference system propagator algorithm (RESPA) scheme ${ }^{22}$ using the
PINY-MD package ${ }^{23}$ with an outer timestep of 4 fs. The bond stretch and bend interactions within molecules were integrated with a timestep of $0.5 \mathrm{fs}$, while the torsional degrees of freedom were handled at 1 fs intervals. The nonbonded interactions were split into two ranges, and the short-range interactions were integrated at 2 fs intervals. Coulombic interactions were treated using the particle mesh Ewald method. The system was initially equilibrated under constant pressure conditions, to arrive at a "natural" simulation box size of linear dimensions $81.4,81.4$, and $52.6 \AA$ at $350 \mathrm{~K}$. The system was equilibrated for around $0.5 \mathrm{~ns}$, and the micelle was found to be stable, with a total pressure of around $100 \mathrm{~atm}$. at $350 \mathrm{~K}$. The analyses reported here were carried out from different sections of a subsequent 3 ns trajectory, during which coordinates of all atoms were stored periodically with a resolution of 1 ps.

The dipolar orientational time correlation function of the water molecules is defined as usual by the following relation:

$$
C_{\mu}(t)=\frac{\left\langle\mu_{i}(t) \cdot \mu_{i}(0)\right\rangle}{\left\langle\mu_{i}(0) \cdot \mu_{i}(0)\right\rangle},
$$

where $\mu_{i}(t)$ is the dipole moment vector of the $i$ th water molecule at time $t$, and $\langle\cdots\rangle$ denotes averaging over initial times 0 , and over water molecules.

The solvation dynamics is monitored by the decay of solvation time correlation function $C_{s}(t)$, which is defined $\mathrm{as}^{2}$

$$
C_{s}(t)=\frac{\langle\delta E(t) \delta E(0)\rangle}{\langle\delta E(0) \delta E(0)\rangle},
$$

where $\delta E(t)$ denotes the fluctuation in the polar part of the potential energy of the tagged cesium ion at time $t$, and the angular bracket is an equilibrium average over different reference zero times, and over cesium ions.

$C_{s}(t)$ was calculated by considering only the polar part of interaction energy, both of a tagged water molecule and of a tagged cesium ion with the rest of the system. Typically, the coordinates of all the atoms from a molecular dynamics run were stored at periodic intervals from which the correlation functions were calculated. For the calculation of $C_{s}(t)$, the polar energy was calculated using a real-space molecular interaction cutoff of $23 \AA$, with no Ewald sum. Note that our molecular dynamics simulations were performed with converged Coulomb energies, using the Ewald summation method, and that we used a simple real-space cutoff during data analysis only. The details are discussed elsewhere. ${ }^{14}$ The solvation energy of a tagged cesium ion consists of contributions from cesium ions, the water molecules, and the polar head groups of the surfactant. Thus, the total solvation time correlation function can have contributions from six distinct partial time correlation functions (three pure and three cross correlations).

Molecules and ions were classified as near $\mathrm{x} \AA$ if they stayed within $\mathrm{x} \AA$ distance from any head group throughout a specified time period. Thus, both the distance and the time duration criteria specify the molecules that qualify for analyses. Far $\mathrm{x} \AA$ molecules were those that stayed beyond $\mathrm{x} \AA$ away from any head group throughout a specified time period. The cutoff distances for classifying molecules and ions 
as near or far were chosen after studying their pair correlation functions with the head group carbon. Another consideration was to obtain a fair number of molecules or ions that can satisfy the rigorous criterion of residence within a layer throughout a particular run (the lengths are defined in captions to the figures). The first neighbor shell of a head group consists of cesium ions and water molecules. Since the counterions and water molecules are in constant exchange between different coordination shells of the head group, a distance cutoff based only on the position of the minimum in the first peak of the pair correlation function might yield only a few (or none) ions or molecules, because of the strict condition of allowing only those molecules which stay in that region throughout a particular time period. Thus, a definition of different interfacial layers based on positions of coordination shells is not quite useful for water molecules which have considerable mobility in the direction perpendicular to the local micellar surface. Wherever possible, we have employed realistic cutoffs for interfacial layers based on the first coordination shell around the head group. Also to note is the fact that different dynamical quantities exhibit different time scales. Thus, to obtain good statistics, one will have to employ different residence time and distance criteria to study these quantities. This aspect has also been discussed before. $^{14}$

The initial configuration for the $300 \mathrm{~K}$ run was obtained from the final configuration of the higher temperature run. Initial MD runs at $300 \mathrm{~K}$ were performed at constant pressure conditions at a set pressure of $1 \mathrm{~atm}$. The density increase at $300 \mathrm{~K}$ relative to the value at $350 \mathrm{~K}$ was found to be $3.1 \%$. The configuration obtained at the end of the constant pressure run was further equilibrated at $300 \mathrm{~K}$ for over $2 \mathrm{~ns}$, under constant volume conditions, after which analyses were carried out over a time period of $4 \mathrm{~ns}$. The average pressure of the system during this constant volume run at $300 \mathrm{~K}$ was found to be $60 \mathrm{~atm}$. It is pertinent to note here that the average size of the micelle increases with decreasing temperature, ${ }^{16}$ with an aggregation number of around 200 at $300 \mathrm{~K}$. It is just impossible for our simulations to capture this growth without an enormous increase in the number of surfactant and water molecules, and thus the computing time. In this work, we assume that the temperature dependence of water and of ion dynamics will not be unduly influenced by the growth of the micelle. Charitably put, our aim in this work is to characterize the effect of temperature alone on the dynamics of the solvent and of the counterions. Indeed, the effects of a larger micelle are likely to affect small molecule or ion dynamics only in the time scales of large amplitude motion of the micelle, such as its rotation. Such "modes" might be expected to operate in the nanosecond time scales, and are thus beyond the scope of this computation. The use of a smaller micelle is less likely to affect the dynamics in the tens to hundreds of picosecond time scales that we are interested in here. Further, in reality, there exists a distribution of micellar sizes in solution at a given temperature and concentration of the surfactant. The so-called aggregation number is just the mean of this distribution. Our study at 300 $\mathrm{K}$ can thus be interpreted as relevant to studying the water

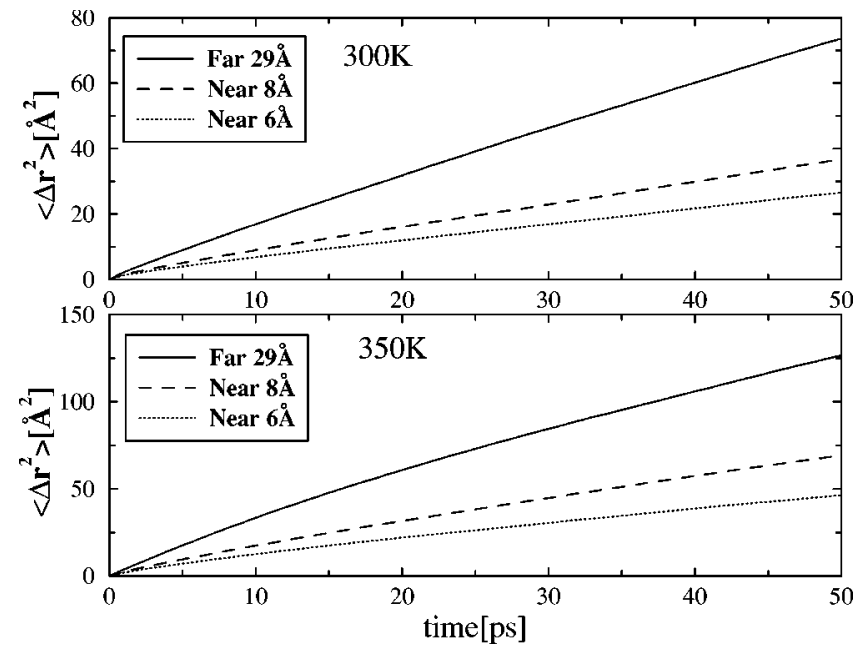

FIG. 1. Mean-square displacement for water molecules near to and far away from the micellar head groups at 300 and $350 \mathrm{~K}$. Near molecules are those which stay within the specified distance from any head group over a time period of $100 \mathrm{ps,}$ and far are those which stay beyond the specified distance for the same time period.

and ion dynamics around micelles at the lower end of this size distribution.

The analyses reported here are made nontrivial by the constant exchange of the probes (water molecules or cesium ions) between different interfacial layers away from the micellar surface. An additional point to note is that the micelle itself is highly fluxional and its surface exhibits dynamical and positional fluctuations, making calculations such as ours quite complex to perform.

\section{RESULTS AND DISCUSSION}

\section{A. Translational diffusion of water at micellar surface}

We have calculated the mean-square displacement of water molecules at different distances away from the micellar surface. These are shown for both temperatures in Fig. 1. The translational diffusion of water molecules is definitely restricted, but the extent of immobility is not dramatic, even at distances as close as $6 \AA$ from the surface. It might well be that only a certain fraction of the water molecules is "bound" to the micellar head groups. At a temperature of $350 \mathrm{~K}$, we have found water molecules to stay within a $10 \AA$ shell from the head group up to about $300 \mathrm{ps,} \mathrm{and} \mathrm{no} \mathrm{longer.}$ This gives us an idea of the lifetime of bound water molecules. In Table I, we provide the diffusion coefficients obtained from the Einstein relation ${ }^{24}$ using the mean-squared

TABLE I. Diffusion coefficients obtained from the long time behavior of the mean-square displacement of water molecules shown in Fig. 1 at two temperatures.

\begin{tabular}{lccc}
\hline \hline & \multicolumn{2}{c}{$\mathrm{D}\left[10^{-5} \mathrm{~cm}^{2} \mathrm{~s}^{-1}\right]$} & \\
\cline { 2 - 3 } & $350 \mathrm{~K}$ & $300 \mathrm{~K}$ & $E_{A}[\mathrm{kcal} / \mathrm{mole}]$ \\
\hline Near 6 & 1.3 & 0.8 & 2.0 \\
Near 8 & 2.0 & 1.2 & 2.4 \\
Far 29 & 3.5 & 2.3 & 1.8 \\
\hline \hline
\end{tabular}


displacement data between the time duration, 30 and $50 \mathrm{ps}$. To obtain this, we have assumed that the diffusion of water molecules takes place in three dimensions, although such an assumption might be questionable for the diffusion of water molecules near the micellar surface, where they will be in a heterogeneous and anisotropic environment. The MSD data in this time range was reasonably linear, and within the statistics available here, we could not identify any nonBrownian diffusion in which the displacement is expected to show a power law behavior. ${ }^{25}$ The coefficient values for water molecules far from the micellar surface match well with values known for bulk SPC/E water reported earlier. ${ }^{26}$ It is interesting to note that that the dimensionless ratio, $\left[D_{\text {far }}(T)-D_{\text {near }}(T)\right] /\left[D_{\text {far }}(T)\right]$, is nearly identical for the two temperatures, suggesting that the extent of restriction in translational diffusion is almost independent of temperature. Assuming an Arrhenius dependence of the diffusion constant with temperature, we find the activation energy to be in the range of $2.0 \mathrm{kcal} / \mathrm{mole}$, and nearly independent of the location of the water molecule. This implies that the additional source of friction on translational motion of the water molecules (as the surface is approached) could have weak temperature dependence. Now, there are at least two types of interactions which are different at the surface. The formation of the hydrogen bond with the polar head groups of the micelle certainly slows down the water molecules, but these bonds (as discussed below) may not be as strong as the bonds with other water molecules. Also, the long-range polar interactions with the other charges of the micelle could have a weaker temperature dependence. The limited set of temperature data precludes us from drawing any conclusions on the nature of the diffusion process near the micellar surface, or whether the diffusion process has a temperature dependence other than the Arrhenius form.

\section{B. Orientational dynamics of surface water}

Unlike translational diffusion, the reorientational motion of water molecules is found to be severely affected near the micellar interface. In Fig. 2, we show the dipolar orientational correlation function $C_{\mu}(t)$ against time for individual water molecules located close to the micellar surface, again for the two temperatures. The data for both show the emergence of a very slow decay at long times, and are nonexponential. In analogy with the relaxation of supercooled liquids, several groups have tried to describe the relaxation near protein surfaces using the stretched exponential function. ${ }^{25,27}$ A fit of our data to a single stretched exponential form was not very satisfactory, and yielded an exponent $(\beta)$ that was 0.24 and 0.36 with a time constant of 2.8 and 10.7 ps at 350 and $300 \mathrm{~K}$, respectively. The low exponent values provide an indication of the extent of nonexponentiality of the relaxation. However, we could satisfactorily fit the dipolar time correlation function (tcf) data to a sum of two stretched exponential functions. The two stretched exponential functions were assumed to describe the limiting behavior, i.e., for water molecules very near the interface (near $6 \AA$ ), and for those far from the interface ( $\operatorname{far} 29 \AA$ ). Thus, we derived two time constants and two exponents, $\beta$. Using these parameters, we were able to fit the dipolar tcf for all intermediate

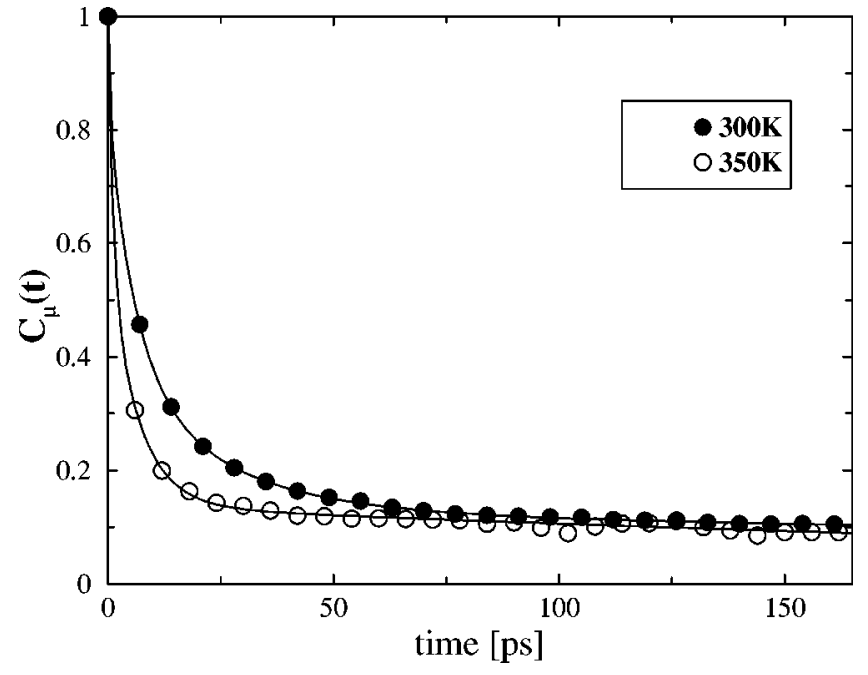

FIG. 2. Dipolar time correlation function, $C_{\mu}(t)$, for water molecules at 300 $\mathrm{K}$ and at $350 \mathrm{~K}$. Only those water molecules which stay within $10 \AA$ from any head group over a time period of 330 ps are used. Continuous line is multiexponential fit at the two temperatures. Numerical data points are shown infrequently for clarity.

distance criteria, with different ratios of mixing. These amplitudes are shown in Table II. The amplitude of one of the stretched exponential form increases as one approaches the surface, indicating the heterogeneous environment that a water molecule experiences. This heterogeneity in the local environment leads to a relatively small $\beta_{1}$ value of around 0.4 . This shows that the distribution of relaxation times near the interface is rather broad. The value of $\beta_{1}$ obtained here is smaller than the value of 0.63 obtained for water molecules near the surface of a protein. ${ }^{25}$ This is somewhat surprising, as the protein possesses a wider variety of surface groups than the single type of surfactant considered here. The latter is thus expected to exhibit a more homogeneous environment for the neighboring water molecules. However, the lower $\beta$ value for the micellar aggregate could have arisen from a greater degree of geometrical disorder over the protein, as

TABLE II. Amplitudes of the two stretched exponential contributions to the dipolar time correlation function of water molecules presented in Fig. 2. The function was fitted to a form: $C_{\mu}(t)=C_{0} \exp \left[-\left(t / \tau_{1}\right)^{\beta_{1}}\right]+C_{1} \exp \left[-\left(t / \tau_{2}\right)^{\beta_{2}}\right]$. The near $6 \AA$ and far $29 \AA$ data were fitted to single stretched exponential functions, respectively, to obtain the following time constant and $\beta$ parameters: $\tau_{1}=9.76 \mathrm{ps}, \beta_{1}=0.43, \tau_{2}=1.84 \mathrm{ps}, \beta_{2}=0.87$ at $350 \mathrm{~K}$, and $\tau_{1}$ $=34.77 \mathrm{ps}, \beta_{1}=0.39, \tau_{2}=3.95 \mathrm{ps}, \beta_{2}=0.80$ at $300 \mathrm{~K}$. Here, $\tau_{1}$ and $\beta_{1}$ correspond to the near $6 \AA$ data and $\tau_{2}$ and $\beta_{2}$ correspond to the far $29 \AA$ data.

\begin{tabular}{cccc}
\hline \hline Temperature [K] & Distance criterion & $C_{0}$ & $C_{1}$ \\
\hline \multirow{3}{*}{350} & Near $6 \AA$ & 1.00 & $\ldots$ \\
Near $8 \AA$ & 0.72 & 0.28 \\
& Near $10 \AA$ & 0.54 & 0.46 \\
& Near $12 \AA$ & 0.43 & 0.57 \\
& Far 29 & $\ldots$ & 1.00 \\
\hline \multirow{3}{*}{300} & Near $6 \AA$ & 1.00 & $\ldots$ \\
& Near $8 \AA$ & 0.70 & 0.30 \\
& Near $10 \AA$ & 0.53 & 0.47 \\
& Near $12 \AA$ & 0.42 & 0.58 \\
& Far 29 & $\ldots$ & 1.00 \\
\hline \hline
\end{tabular}


TABLE III. Parameters of multiexponential fits to the dipolar time correlation function of water molecules presented in Fig. 2 at two temperatures.

\begin{tabular}{|c|c|c|c|c|}
\hline & \multicolumn{2}{|c|}{$350 \mathrm{~K}$} & \multicolumn{2}{|c|}{$300 \mathrm{~K}$} \\
\hline & $\tau[\mathrm{ps}]$ & Amplitude & $\tau[\mathrm{ps}]$ & Amplitude \\
\hline & 1.1 & 0.42 & 0.5 & 0.17 \\
\hline & 6.4 & 0.44 & 6.0 & 0.44 \\
\hline & $\ldots$ & $\ldots$ & 22.8 & 0.26 \\
\hline & 383.5 & 0.14 & 767.1 & 0.13 \\
\hline$\tau_{\mu}[\mathrm{ps}]$ & \multicolumn{2}{|c|}{57} & \multicolumn{2}{|c|}{108} \\
\hline
\end{tabular}

well as from the fact that we have chosen to study the reorientation of only those water molecules with long residence times in the hydration layer. This could lead to the slower decay observed in our simulations than in the work of Rocchi et al. ${ }^{25}$ Another reason could be that the binding energies near uncharged, polar groups in proteins are around 1.4 $\mathrm{kcal} / \mathrm{mole},{ }^{28}$ while the binding energy for the micelle studied here is at least $50 \%$ larger than the former. This could lead to a lower exponent for the stretched exponential function. The observation that the reorientational time correlation function can be fitted to a sum of two functions can also be rationalized from the two-state dynamical equilibrium model. ${ }^{12}$ Fits to a sum of simple and stretched exponential form were not as satisfactory as the fits to a sum of two stretched exponential forms.

It is also useful to fit the dipolar tcf to a multiexponential form rather than to a sum of stretched exponential functions. The former is attractive, as one can directly obtain an average time constant, which can provide us information on the extent of slowdown in the water and ion dynamics. This approach is also widely used by experimentalists. Further, it is in principle possible to assign the different time constants to different relaxation processes of the system. For instance, the ultrafast decay observed in the water reorientation can be related to its fast librational motion. It is in this spirit that we have chosen to fit the dipolar tcf data to a sum of simple exponential functions. The time constants obtained by fitting to such a multiexponential form are shown in Table III, where we have also given the value of the average orientational correlation time, $\tau_{\mu}$, defined by

$$
\tau_{\mu}=\int_{0}^{\infty} d t C_{\mu}(t) .
$$

From Fig. 2 and Table III, we can reach the following conclusions:

(a) The decay of the orientational correlation function is nonexponential at both temperatures. One needs at least four exponentials to fit the data at $300 \mathrm{~K}$.

(b) The average orientational correlation time is slower by about a factor of 20 than the corresponding value in the bulk. However, the long time decay is slower by more than two orders of magnitude. Such slowdown has been observed in real experiments.

(c) The effects of the heterogeneous surface on the orientational dynamics is rather dramatic at $300 \mathrm{~K}$. Now, almost $40 \%$ of the decay falls under the slow region. This graph is perhaps the first demonstration of such a dramatic slowing down of orientational dynamics near a surface which interacts strongly with the water molecules. The time constants for this decay are also given in Table III.

(d) We see from Fig. 2 that $C_{\mu}(t)$ for water molecules near the micellar interface does not decay to zero, as opposed to its decay to zero for water molecules in bulk. Also note that the function exhibits a nearly constant value at long times, spanning over 100 to $200 \mathrm{ps}$. The decay to zero of this function for bulk water is a consequence of the water dipole being able to explore all possible orientations in space. For water molecules near an interface, this exploration is restricted to a cone, due to the fact that they are hydrogen bonded to the micellar head groups, and that micellar dynamics is much slower. Thus, the long time value of this function is a direct indicator of the extent of this restriction, with larger values signifying a more restricted environment for molecular reorientation. Note that the function does not remain constant, and does actually decay, albeit at a very slow rate. This decay arises due to the unshackling of the water molecule from the cone due to the breaking of the hydrogen bonds that it had formed with the polar head groups of the surfactants. ${ }^{29}$

The orientational correlation will of course decay in the very long time (of the order of tens or hundreds of ns), either due to "evaporation" of the slow water molecules at the surface or due to rotation of the micelle itself. Note that this slow orientational decay has already been observed in experiments, as discussed earlier.

It is now natural to ask if the reorientation of water is hindered or slowed down within this cone. This information can be obtained by studying the time correlation function of the fluctuation in dipole moment, rather than the dipole moment itself. This function is defined as

$$
C_{\mu, i}^{\prime}(t)=\frac{\left\langle\delta \mu_{i}(t) \cdot \delta \mu_{i}(0)\right\rangle}{\left\langle\delta \mu_{i}(0) \cdot \delta \mu_{i}(0)\right\rangle},
$$

where $\delta \mu_{i}(t)=\mu_{i}(t)-\left\langle\mu_{i}\left(t^{\prime}\right)\right\rangle$ is the fluctuation in the dipole moment, and the average in the second term is taken over all times, $t^{\prime}$. This quantity is plotted for water molecules near the micellar surface at 350 and at $300 \mathrm{~K}$ in Fig. 3, and is compared to the function for water molecules in the far region around the micelle. The reorientation of water molecules near the interface is not only restricted to a cone, but the motion within this cone is also considerably slowed down, relative to water molecules in the bulk. The time correlation functions for near water in this figure could be fitted to two exponentials with time constants of 0.4 and 2.4 ps at $350 \mathrm{~K}$ and 0.9 and 5.4 ps at $300 \mathrm{~K}$, respectively. In both cases, the faster component contributes about $25 \%$ of the total decay. The average time constant obtained for water molecules close to the surface is about a factor of 2 larger than that for water molecules in the bulk region. This shows conclusively that the molecular reorientation is definitely slowed down near the micelle.

From the values of the time constant of the slowest component in the orientational relaxation (Fig. 2) at the two tem- 


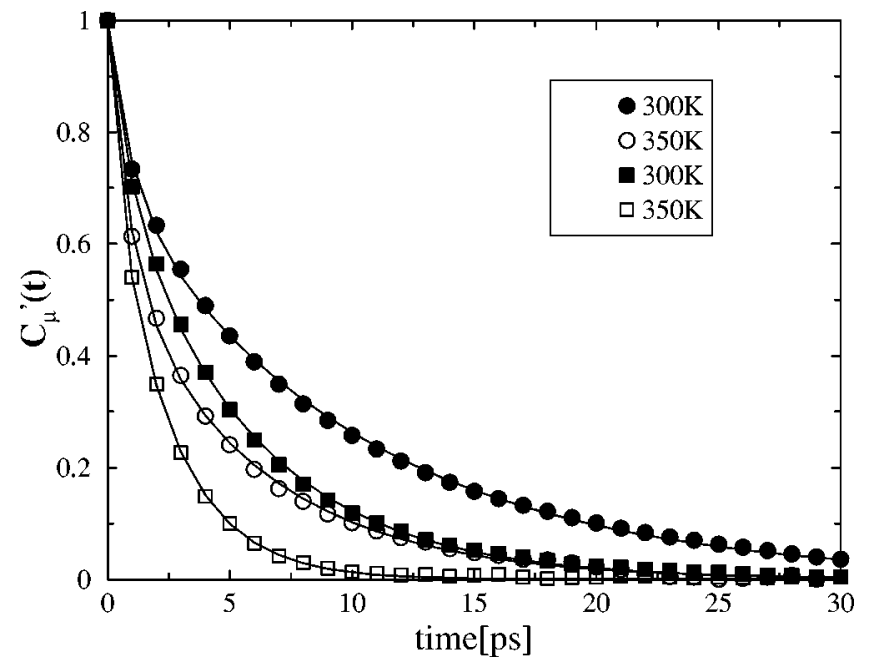

FIG. 3. Time correlation function of fluctuations, $C_{\mu}^{\prime}(t)$, in water dipole for water molecules at $300 \mathrm{~K}$ and at $350 \mathrm{~K}$. Circles are for those water molecules which stay within $10 \AA$ from any head group over a time period of $330 \mathrm{ps}$, while the squares are for those water molecules which are beyond 29 $\AA$ away from any head group. The continuous line is the multiexponential fit at the two temperatures for the waters near the head groups. Numerical data points are shown infrequently for clarity.

peratures, we can calculate an activation energy for the rotational diffusion, which we found to be $2.9 \mathrm{kcal} / \mathrm{mole}$. This value is smaller than the hydrogen bond-breaking energy for pure water in the bulk. However, it is known that the hydrogen bond energies of water with biological systems vary between 1.0 to $2.8 \mathrm{kcal} / \mathrm{mole}^{28}$ Thus, the value $2.9 \mathrm{kcal} / \mathrm{mole}$ seems reasonable. We have already discussed that the slowing down may originate partly from the hydrogen bond bridge that a water molecule at the surface of the CsPFO micelle can form with the neighboring polar head groups. ${ }^{15}$ Thus, the rotation of the water molecule may involve breaking of one or two bonds. Here, we provide some more structural data in addition to what has been published earlier. ${ }^{15}$ Figure 4(a) shows the pair correlation function, $g(r)$ between the head group oxygen and the oxygen atom of the water molecule at $300 \mathrm{~K}$. The $g(r)$ at $300 \mathrm{~K}$ is nearly identical to the one at $350 \mathrm{~K}$ but has sharper features; hence, the latter is not shown here. The first coordination shell is at a
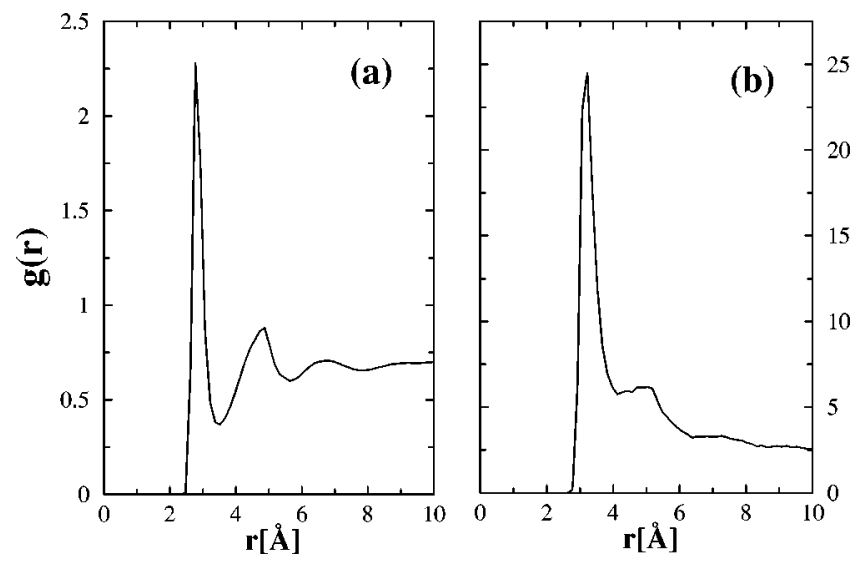

FIG. 4. Pair correlation functions at $300 \mathrm{~K}$ for (a) head group oxygen-water oxygen, and (b) head group oxygen-cesium pairs.

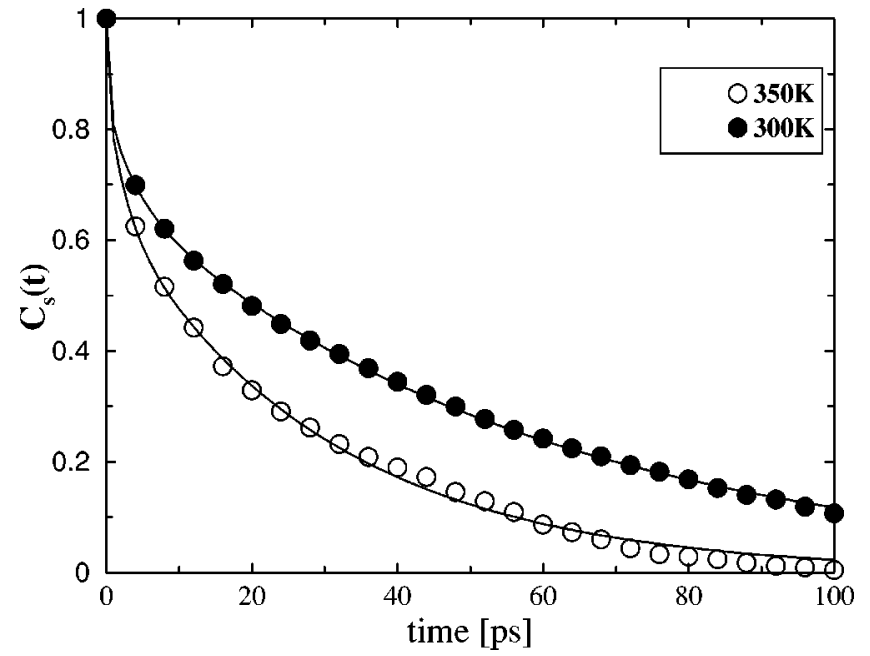

FIG. 5. The decay of the solvation time correlation function, $C_{s}(t)$, for cesium ions at 300 and $350 \mathrm{~K}$. The solvation time correlation function is shown for only those $\mathrm{Cs}^{+}$ions which stay within $10 \AA$ from any head group for $1 \mathrm{~ns}$. Continuous line is multiexponential fit at the two temperatures. Numerical data points are shown infrequently for clarity.

distance of $2.90 \AA$, with a coordination number of around 3.5. This is consistent with our earlier observations ${ }^{15}$ that the coordination number of water molecules around the head group carbon at $350 \mathrm{~K}$ is 6.3 . In Fig. 4(b), we provide the $g(r)$ between headgroup oxygens and cesium ions at $300 \mathrm{~K}$. One finds that the cesium ions have nearly "condensed" on the surface of the micelle, indicated by a strong peak at 3.20 $\AA$, with a coordination number of 0.44 . This, too, is consistent with our earlier observations from an analysis of the head group carbon-cesium $g(r)$ that nearly $70 \%$ of the cesium ions present in the system is present in the first coordination shell of the micellar surface. ${ }^{14,30}$

It should be remarked here that another source of rotational slowing down may come from the long-range polar interactions of a single water molecule with the other charge groups of the micelle. Since the motion of the micelle is very slow compared to the motion of water molecules, this friction may be significant. Actually, this motion could have been significant if not for the fast motion of the water molecules themselves.

\section{Solvation dynamics}

For solvation dynamics of dipoles in pure water, experimental studies find a dominant sub-50 fs Gaussian component and a slow biexponential decay with time constants 126 and $880 \mathrm{fs}$, respectively. ${ }^{31-34}$ It is believed that the initial ultrafast response comes from the intermolecular $\mathrm{O} \cdots \mathrm{O}$ vibrational mode of water, while the slowest one comes from the reorientational dynamics of water. ${ }^{34,35}$ As the latter is most affected by the presence of the micellar surface, we expect the slower component of solvation dynamics to be really affected and exhibit strong temperature dependence.

In Fig. 5 we show the solvation dynamics of cesium ions near the micellar surface at 300 and $350 \mathrm{~K}$. Note the strong temperature dependence. The slow tail in the decay of $C_{s}(t)$, already clear at $350 \mathrm{~K}$ (and reported earlier ${ }^{14}$ ), becomes more 
TABLE IV. Parameters of multiexponential fits to the solvation time correlation function of cesium ions presented in Fig. 5 at two temperatures.

\begin{tabular}{|c|c|c|c|c|}
\hline & \multicolumn{2}{|c|}{$350 \mathrm{~K}$} & \multicolumn{2}{|c|}{$300 \mathrm{~K}$} \\
\hline & $\tau[\mathrm{ps}]$ & Amplitude & $\tau[\mathrm{ps}]$ & Amplitude \\
\hline & 0.2 & 0.15 & 0.3 & 0.15 \\
\hline & 2.6 & 0.20 & 3.9 & 0.15 \\
\hline & 30.0 & 0.65 & 56.4 & 0.70 \\
\hline$\tau_{s}[\mathrm{ps}]$ & \multicolumn{2}{|c|}{20} & \multicolumn{2}{|c|}{40} \\
\hline
\end{tabular}

important at $300 \mathrm{~K}$. The time constant of the long time tail is around $30 \mathrm{ps}$ at $350 \mathrm{~K}$ and $56 \mathrm{ps}$ at $300 \mathrm{~K}$. We have fitted the decay of the solvation time correlation function to multiexponential forms in both the cases; the values of the time constants and the relative amplitudes of the different time constants are presented in Table IV. As before, one can make the following observations from the fits:

The decay of the solvation time correlation function is also nonexponential at both the temperatures. One needs at least three exponentials to fit the data at 350 and $300 \mathrm{~K}$.

(ii) The average solvation time [defined by $\tau_{s}$ $\left.=\int_{0}^{\infty} d t C_{s}(t)\right]$ is slower by about a factor of 20 than the corresponding value in the bulk. However, the long time decay is slower by more than two orders of magnitude. Such a slowdown has been observed in real experiments. ${ }^{10}$

(iii) The slow components contribute to almost $65 \%$ to $70 \%$ of the decay of the solvation time correlation function. This graph is perhaps also the first demonstration of such a dramatic slowing down of solvation dynamics near a surface which interacts strongly with the water molecules.

There is an important distinction to be made in terms of residence times between water molecules and cesium ionsthe latter have significantly longer residence times near the micellar interface than the former. At $300 \mathrm{~K}$, the cesium ions nearly "condense" on top of the micelle, to presumably form the Stern layer. This gives us a much wider time window to study their long time dynamics, which was not possible in the case of water molecules, due to their relatively rapid exchange between interfacial layers.

The time constants of the slow decay that we have obtained from the reorientational motion of water molecules and that from the solvation dynamics of cesium ions are significantly different, with the latter seemingly exhibiting a smaller time constant. We believe that this difference could come from the choice of the "probe" that we have used in solvation dynamics, i.e., the cesium ion. The cesium ions are reasonably heavy particles, but still could have significant translational motion in the time scales of 50 to 100 ps. A good probe of solvation dynamics should be stationary, so that the solvation response comes purely from the surrounding solvent. ${ }^{35}$ It is quite possible that if we use a larger probe molecule with less mobility, as in experiments, we might observe a much slower decay in solvation dynamics.

We have demonstrated earlier from an analysis of the partial solvation time correlation functions for the micellar system that the contribution from the polar interaction of the cesium ion with the micelle head groups is the most dominant cause of the slow decay. ${ }^{14}$ In contrast to this partial tcf, the contribution from the interaction with water molecules is much less dominant. Thus, the slow part of the polar solvation of the cesium ions may have more to do with the hydrogen bond-breaking dynamics between PHG of the micelles and the water molecules. ${ }^{29}$ The bond-breaking dynamics is connected not only with the dynamics of the surrounding water molecules at the micellar surface but also with the micellar dynamics. Thus, the slow solvation and slow orientational relaxation at the surface are indeed probes of interaction of water molecules with the micellar surface.

In the simulations reported here, we have taken adequate care to calculate the time correlation functions over times much longer than the relaxation times of the functions. Due to the heterogeneous nature of the hydrophilic surface, all the ions or molecules that satisfy the residence criteria in the interfacial layer need not be in similar environments. Hence, in the calculation of time correlation functions of fluctuating quantities, we have subtracted the time average of the quantity of interest corresponding to each particle separately [as in Eq. (4)], instead of subtracting the average over both time and particles. The latter type of averaging might lead to quantitatively erroneous results in heterogeneous environments.

\section{CONCLUSIONS}

The present simulations show a strong temperature dependence of the orientational relaxation of water molecules and also of the solvation dynamics of ions at the micellar surface. As noted earlier, a strong temperature dependence is expected because the micelle is formed and held together by soft interactions like van der Waals and hydrogen bonding. In addition, these interactions act collectively to stabilize the micelle. The appearance of the very slow component at 300 $\mathrm{K}$ in the dipolar orientational correlation function, with a relaxation time that is two orders of magnitude slower than that in the corresponding bulk solvent, is certainly a main result of this study. This aspect seems to be in agreement with the experimental results, although experimentally one studies the dipolar solvation dynamics of a large dye molecule near such surfaces, while our simulations focus on ionic solvation. Another difference between experiments and our simulations has to do with averaging. Our simulations contain only one micelle, whereas experimentally one studies the average response from several dye molecules in a micellar solution.

The present study shows that the slowing down of the reorientational motion of water arises from the formation of bridge hydrogen bonds that water molecules form with polar head groups of the micelle. The two hydrogen bonds of water seem to form a bridge between the neighboring polar head groups. Such bridges have been observed in hydrated jet-cooled biomolecules, ${ }^{36}$ and also in MD simulations of $\mathrm{C}_{12} \mathrm{E}_{2}$ surfactants in water. ${ }^{37}$ Such bridges, once formed, gain stability from the cooperative strengthening of the bonds. 
Here, we show that these strengthening leads to slow down of the rotation of water molecules.

The reorientational motion and diffusion of water molecules in different hydration shells was earlier examined in micelles of sodium octanoate using the molecular dynamics method by Watanabe and Klein. ${ }^{20}$ In the time scales of a few picoseconds, they also observed slow reorientational and translational dynamics of water near the micelle. Recently, Faeder and Ladanyi ${ }^{38}$ reported results of MD simulations of model reverse micelles taking into account many experimental quantities explicitly. They found that both the translational and the rotational mobility of the trapped and bound water were significantly slower than that of free water. Interestingly, their preliminary results did not indicate significant slowing down of the solvation dynamics in the water pool, which is in disagreement with the present study. ${ }^{39}$ It would be interesting to compare the results of the two simulations, especially the role of the partial time correlation functions.

The appearance of the slow component in solvation dynamics of ions, particularly at $300 \mathrm{~K}$, is due primarily to their interaction with the micelle. Our simulations point to the dominant role of the micelle as the reason for the slow dynamics. This feature merits further attention, and might require simulations of other micellar systems. Our simulations show that micellar dynamics gets seriously restrained at ambient conditions, which leads to a further slowing down of water reorientation.

Further studies will concentrate on the lifetime of hydrogen bonds near the surfactant surface. We are also investigating detailed water trajectories to understand the transition between the bound and the free water molecules. Another point of interest is the role of the slow relaxation on the chemical reactions at the micellar surface. It is clear that the rates of electron transfer reactions can be affected by this slow component. ${ }^{11}$ Work in these directions is under progress.

\section{ACKNOWLEDGMENTS}

The research reported here was supported in part by grants from the Council of Scientific and Industrial Research (CSIR) and the Department of Science and Technology (DST), Government of India.

\footnotetext{
${ }^{1}$ See for example, "Hydration processes in biological and macromolecular systems," Faraday Discuss. 103, 1 (1996).

${ }^{2}$ N. Nandi, K. Bhattacharyya, and B. Bagchi, Chem. Rev. 100, 2013 (2000).

${ }^{3}$ M. Fukuzaki, N. Miura, N. Sinyashiki, D. Kunita, S. Shiyoya, M. Haida, and S. Mashimo, J. Phys. Chem. 99, 431 (1995); P. S. Belton, ibid. 99, 17061 (1995).

${ }^{4}$ N. Sarkar, A. Datta, S. Das, and K. Bhattacharyya, J. Phys. Chem. 100,
}

15483 (1996); A. Datta, D. Mandal, S. K. Pal, and K. Bhattacharyya, J. Mol. Liq. 77, 121 (1998).

${ }^{5}$ X. J. Jordanides, M. J. Lang, X. Song, and G. R. Fleming, J. Phys. Chem. 103, 7995 (1999); N. Nandi and B. Bagchi, ibid. 102, 8217 (1998).

${ }^{6}$ R. E. Riter, D. M. Willard, and N. E. Levinger, J. Phys. Chem. B 102, 2705 (1998).

${ }^{7}$ N. E. Levinger, Curr. Opin. Colloid Interface Sci. 5, 118 (2000).

${ }^{8}$ B. Halle, V. P. Denisov, and K. Venu, in Biological Magnetic Resonance, edited by N. Ramakrishna and L. J. Berliner (Kluwer Academic/Plenum, New York, 1999), Vol. 17, p. 419; V. P. Denisov, J. Peters, H. D. Horlein, and B. Halle, Nat. Struct. Biol. 3, 505 (1996).

${ }^{9}$ G. Otting, in Biological Magnetic Resonance, edited by N. Ramakrishna and L. J. Berliner (Kluwer Academic/Plenum, New York, 1999), Vol. 17, p. 485; G. Otting and E. Liepinsh, Acc. Chem. Res. 28, 171 (1995).

${ }^{10}$ K. Bhattacharyya and B. Bagchi, J. Phys. Chem. 104, 10603 (2000).

${ }^{11}$ L. Chen, P. D. Wood, A. Mnyusiwalla, J. Marlinga, and L. J. Johnston, J. Phys. Chem. B 105, 10927 (2001).

${ }^{12}$ N. Nandi and B. Bagchi, J. Phys. Chem. B 101, 10954 (1997).

${ }^{13}$ K. Hara, H. Kuwabara, and O. Kajimoto, J. Phys. Chem. B 105, 7174 (2001).

${ }^{14}$ S. Balasubramanian and B. Bagchi, J. Phys. Chem. B 105, 12529 (2001).

${ }^{15}$ S. Balasubramanian and B. Bagchi, J. Phys. Chem. B 106, 3668 (2002).

${ }^{16}$ N. Boden, P. H. Jackson, K. McMullen, and M. C. Holmes, Chem. Phys. Lett. 65, 476 (1979); N. Boden, K. W. Jolley, and M. H. Smith, J. Phys. Chem. 97, 7678 (1993); N. Boden, S. A. Corne, M. C. Holmes, P. H. Jackson, D. Parker, and K. W. Jolley, J. Phys. (France) 47, 2135 (1986).

${ }^{17}$ H. Iijima, T. Kato, H. Yoshida, and M. J. Imai, J. Phys. Chem. B 102, 990 (1998).

${ }^{18}$ W. M. Gelbart and A. Ben-Shaul, J. Phys. Chem. 100, 13169 (1996).

${ }^{19}$ H. J. C. Berendsen, J. R. Grigera, and T. P. Straatsma, J. Phys. Chem. 91, 6269 (1987).

${ }^{20}$ K. Watanabe and M. L. Klein, J. Phys. Chem. 93, 6897 (1989); 95, 4158 (1991).

${ }^{21}$ M. Sprik, U. Röthlisberger, and M. L. Klein, Mol. Phys. 97, 355 (1999).

${ }^{22}$ M. E. Tuckerman, B. J. Berne, and G. Martyna, J. Chem. Phys. 97, 1990 (1992).

${ }^{23}$ M. E. Tuckerman, D. A. Yarne, S. O. Samuelson, A. L. Hughes, and G. Martyna, J. Comput. Phys. Commun. 128, 333 (2000).

${ }^{24}$ M. P. Allen and D. J. Tildesley, Computer Simulation of Liquids (Clarendon, Oxford, 1987).

${ }^{25}$ C. Rocchi, A. R. Bizzarri, and S. Cannistraro, Phys. Rev. E 57, 3315 (1998).

${ }^{26}$ S. Balasubramanian, C. J. Mundy, and M. L. Klein, J. Chem. Phys. 105, 11190 (1996).

${ }^{27}$ M. Tarek and D. J. Tobias, Biophys. J. 79, 3244 (2000); Phys. Rev. Lett. 88, 138101 (2002).

${ }^{28}$ G. A. Jeffrey and W. Saenger, Hydrogen Bonding in Biological Systems (Springer, New York, 1991), Chap. 2.

${ }^{29}$ S. Balasubramanian, S. Pal, and B. Bagchi, Phys. Rev. Lett. (submitted).

${ }^{30}$ S. Balasubramanian, S. Pal, and B. Bagchi, Curr. Sci. 82, 845 (2002).

${ }^{31}$ R. Jimnez, G. R. Fleming, P. V. Kumar, and M. Maroncelli, Nature (London) 369, 471 (1994)

${ }^{32}$ M. Maroncelli and G. R. Fleming, J. Chem. Phys. 89, 5044 (1998).

${ }^{33}$ M. S. Skaf and B. M. Ladanyi, J. Phys. Chem. 100, 18258 (1996).

${ }^{34}$ S. Roy and B. Bagchi, J. Chem. Phys. 99, 9938 (1993); N. Nandi, S. Roy, and B. Bagchi, ibid. 102, 1390 (1995).

${ }^{35}$ B. Bagchi and R. Biswas, Adv. Chem. Phys. 109, 207 (1999).

${ }^{36}$ T. S. Zwier, J. Phys. Chem. A 105, 8827 (2001).

${ }^{37}$ S. Bandyopadhyay, M. Tarek, M. L. Lynch, and M. L. Klein, Langmuir 16, 942 (2000).

${ }^{38}$ J. Faeder and B. M. Ladanyi, J. Phys. Chem. B 104, 1033 (2000).

${ }^{39}$ J. Faeder and B. M. Ladanyi, J. Phys. Chem. B 105, 11148 (2001). 